\title{
Spatio-temporal expression of chromogranin A during zebrafish embryogenesis
}

\author{
Jing Xie ${ }^{1, *}$, Wei-Qing Wang ${ }^{1, *}$, Ting-Xi Liu ${ }^{2,3}$, Min Deng ${ }^{3}$ and Guang Ning ${ }^{1}$ \\ ${ }^{1}$ School of Medicine, Shanghai Institute of Endocrinology and Metabolism, Shanghai Clinical Center for Endocrine and Metabolic Disease and ${ }^{2}$ State Key \\ Laboratory for Medical Genomics, School of Medicine, Ruijin Hospital, Shanghai Jiaotong University, 197 Ruijin Road II, Shanghai 200025, People's \\ Republic of China \\ ${ }^{3}$ Laboratory of Development and Diseases, School of Medical, Institute of Health Sciences, Shanghai Institute for Biological Sciences, Chinese Academy of \\ Sciences and Shanghai Jiaotong University, Chongqing South Road 225, Shanghai 200025, People's Republic of China \\ (Correspondence should be addressed to G Ning; Email: guangning@medmail.com.cn) \\ *(J Xie and W-Q Wang contributed equally to this work)
}

\begin{abstract}
Chromogranin A (CHGA), a protein participating in the biogenesis of dense core secretory granules in various neuroendocrine tissues, plays a critical role in the release of hormones/peptides and the pathogenesis of pheochromocytoma. However, little is known about the developmental origin of CHGA-expressing cells during embryogenesis. Here, we report the structural characterization and spatiotemporal expression pattern of zebrafish (Danio rerio) ortholog of mammalian CHGA. The earliest expression of chga transcripts was observed at $16 \mathrm{~h}$ post fertilization in the developing cranial ganglia as six distinct cellular masses arranged bilaterally as strings of beads in the dorsal root ganglia (DRG) precursors along the dorsal trunk. With development advancing, the chga transcripts were expressed abundantly in
\end{abstract}

diencephalon, mesencephalon, and rhombencephalon as well as in the DRG. Interestingly, double in situ hybridization assay of chga with genes expressed in pronephros (Wilms' tumor suppressor 1, wt 1), adrenal cortex (side-chain cleavage enzyme, $s c c$ ), and sympathoadrenal neuron/chromaffin cell (dopamine$\beta$-hydroxylase, $d b h)$, respectively, showed that the chgaexpressing cells are spatially separated from $w t 1^{-}, s c c-$, and $d b h$-positive cell populations during early embryonic development. The pronephros region does not express chga even up to 7 days post fertilization, while chga positive-staining cells bind in the brain and DRG, indicating that chga may play an important role in nervous system development during the early embryonic stages.

Journal of Endocrinology (2008) 198, 451-458

\section{Introduction}

Chromogranin, a family of soluble, acidic proteins being co-secreted with resident hormones and biogenic amines, represents many major constituents in the secretory vesicle cores of virtually all neuroendocrine tissues. Over the past four decades, seven proteins of chromogranin family with distinct functions have been identified: chromogranin A, $\mathrm{B}$, and $\mathrm{C}$ (also known as secretogranin II), and four other acidic secretory proteins, secretogranin III, IV, V, and VI. Chromogranin A (CHGA) was originally identified as a major soluble protein in the chromaffin granules of adrenal medullary four decades ago and has been extensively studied as to its structure, expression, and function (Taupenot et al. 2003). Both in vitro and in vivo experiments have demonstrated that the CHGA plays a critical role in a number of vital physio- and pathological processes, including the biogenesis of dense core secretory granules, immunity against microbes, neurodegenerative disorders, cardiovascular diseases (e.g., hypertension), and as a potential marker for several endocrine tumors, such as pheochromocytoma, paraganglioma, gastrinoma, and insulinoma (Kim \& Loh 2005).

Although the study of CHGA has been explored widely, the expression of CHGA at early stages of development has remained elusive because of the invisibility of the embryogenesis in mammals. Several animal models (chicken, bovine, and rat) have been employed for studying the developmental and anatomic distribution of chga-positive cells in these species. The existing results mainly indicate the expression of CHGA protein in adrenal medulla and gastro-entero-pancreatic system that abounds with various endocrine cells (Kent \& Coupland 1989, Mahata et al. 1993, Wang et al. 1994, Totzauer et al. 1995, Kameda et al. 1998). The distribution of Chga transcripts in the rat brain has also been reported (Mahata et al. 1993).

The zebrafish has become an important vertebrate model for studying human development and endocrine diseases, because of its many unique advantages, such as a large number of embryos per clutch, rapid and external development, 
and optical clarity, which allows for the direct visualization of organogenesis and cellular migration (McGonnell \& Fowkes 2006). In this study, we investigated the zebrafish chga, which is the ortholog of the human counterpart, and performed whole-mount in situ hybridization (WISH) using the anti-sense chga probe to observe the spatiotemporal distribution of chga-positive cells during early development.

In mammals, the adrenal cortex derives from the embryonic mesoderm, in contrast to the ectodermal origin of the medulla. The adrenal glands (with the cortex around the medulla) located at the upper poles of the kidneys at the late stage of embryogenesis, however, continue to develop for years after birth (Unsicker et al. 2005). In fish, things are quite different: in some species, the cortex and the medulla are separated, while in others, they are fused together. In the former ones, the adrenal cortex is located between two kidneys, with the neighboring medulla (with chromaffin cells forming small clusters) spreading toward and adjoining the sympathetic nerves and blood vessels; while in the latter ones, the two kinds of adrenal cells are embedded in the lymphoidlike hematopoietic cells of the head kidney, and this area is called 'pronephros' (Grassi Milano et al. 1997). Zebrafish, a kind of teleost, is confirmed to have an intermingled group of cells named as interrenal in the pronephros that produce both catecholamines (CAs) and steroid hormones in adults (Grassi Milano et al. 1997, Hsu et al. 2003). Although the anatomical positions of the kidneys, adrenal cortex, and medulla in adult fish are explored widely, their early developments remain unknown. Our study aims to find out the origin of CHGApositive cells and the development of the chromaffin cells in embryogenesis. In zebrafish, it is found that the interrenal development parallels that of the embryonic kidney (pronephros; Hsu et al. 2003). The chromaffin cells and interrenal gland cells are mixed together, both of which are situated in the head kidney region of adult kidney (Grassi Milano et al. 1997, Hsu et al. 2003). The chromaffin cells contain vesicles with strong electron-dense granules under transmission electron (Hsu et al. 2003). In human and other species, CHGA is considered to be a relatively specific protein expressed in adrenal medulla chromaffin cells (Tischler $e t$ al. 2004), in zebrafish, side-chain cleavage enzyme $(s c c)$ and Wilms' tumor suppressor 1 (wt 1) are the markers of interrenal (Hsu et al. 2003) and pronephros (Chai et al. 2003, Hsu et al. 2003) respectively. Therefore, it is intriguing to investigate the anatomic relationship between CHGA-expressing cells and the interrenal cells/pronephros during embryogenesis by double in situ hybridization assay.

Furthermore, in humans, dopamine- $\beta$-hydroxylase (DBH) and CHGA, both co-express in the chromaffin cells, are critical for CA biogenesis, storage, and release. According to the previous study, $d b h$ is a marker of sympathoadrenal noradrenergic neurons/chromaffin cells in zebrafish (Reid et al. 1995), and we would also like to find out whether $d b h$ and chga-positive cells are of the same origin.

\section{Materials and Methods}

\section{Fish culture and embryo collection}

Zebrafish adults and embryos were maintained at $28.5{ }^{\circ} \mathrm{C}$ under standard conditions. Staging of embryos was carried out according to Kimmel et al. (1995). Embryos were cultured in $0.03 \%$ phenylthiourea (Sigma) solution starting at $12 \mathrm{hpf}$ to eliminate pigmentation. Zebrafish for the experiment were maintained under the guidelines of the Chinese Council of Animal Care.

\section{Bioinformatics analysis}

The information of the Danio rerio chga protein sequence and that of the other species were obtained from the web sites http://www.ncbi.nlm.nih.gov and http://www.ensembl.org. The database of $D$. reriochga genomic structure was retrieved from the web site http://www.ncbi.nlm.nih.gov. The human and $D$. rerio gene maps were constructed based on gene loci from LocusLink (http://www.ncbi.nlm.nih.gov/LocusLink/). The protein alignment and the bootstrap neighbor-joining phylogenetic tree were constructed with CLUSTAL X using the $D$. rerio chga amino acid sequence as an outgroup (bases on number per 500 duplicates).

\section{Cloning of zebrafish chga}

Reverse transcriptase PCR (RT-PCR) was performed using total RNA isolated from $7 \mathrm{dpf}$ zebrafish embryos. Total RNA was prepared using TRIzol (Life Technologies, Inc.) followed by reverse transcription (RT) according to manufacturer's instruction (Promega). chga mRNA was amplified by La-Taq (TaKaRa, Otsu, Shiga, Japan) and the primers used were as follows: forward 5'- GGGAGTTCAAGCACTCCAAG and reverse $5^{\prime}$ - TTCTGGAAACCCAAAAACCA to obtain a cDNA fragment corresponding to the nucleotides 10491699 bp of the zebrafish chga. Reactions were carried out in a PTC-225 DNA Engine Tetrad (MJ Research Inc., Waltham, MA, USA). Denaturation was performed at $95^{\circ} \mathrm{C}$ for $3 \mathrm{~min}$., followed by $95{ }^{\circ} \mathrm{C}$ for $30 \mathrm{~s}$, annealing at $58^{\circ} \mathrm{C}$ for $30 \mathrm{~s}$ and extension at $72^{\circ} \mathrm{C}$ for $40 \mathrm{~s}$ for 30 cycles. The PCR products were loaded onto $1.2 \%$ agarose gels and visualized by ethidium bromide under u.v. light.

Whole-mount $m R N A$ in situ hybridization and double in situ hybridization

The chga cDNA fragment was cloned into the PCSII+ vector, BamH1 was used to digest the plasmid and T7 RNA polymerase was used for the synthesis of either digoxigeninor fluorescein-labeled zebrafish chga RNA anti-sense probes. As a control, sense RNA was synthesized by Sp6 RNA polymerase. Plasmids for $s c c$ and $d b h$ were linearized with XhoI and $\mathrm{NcoI}$ respectively, and transcribed with Sp6 polymerase. Wt1 plasmid was linearized with Not I and transcribed with 
T7 polymerase. The $s c c$ plasmid and $d b h$ plasmid were kindly provided by Prof. B C Chung (Academia Sinica, Taipei, Taiwan) and Dr V Korzh (Institute of Molecular and Cell Biology, Singapore) respectively. All of the constructs were verified by restriction analysis and nucleotides sequence analysis.

WISH and double in situ hybridization were performed by the protocols according to previous study (Song et al. 2004). Embryos were collected from timed matings, raised at $28.5{ }^{\circ} \mathrm{C}$, and carefully staged before fixing overnight at $4{ }^{\circ} \mathrm{C}$ in $4 \%$ paraformaldehyde (PFA) in PBS. Digoxigenin- or fluorescein-labeled anti-sense RNA and sense RNA probes were generated for chga, $w t 1, s c c$, and $d b h$. Probes were detected using anti-digoxigenin or anti-fluorescein antibodies conjugated to alkaline phosphatase (Roche), followed by incubation with 5-bromo 4-chloro 3-indolyl phosphate and nitro blue tetrazolium or Fast Red.

For analysis of the double in situ hybridization, digoxigenin-labeled signals were captured using transmitted light and fluorescein-labeled signals were captured using an Argon 543-nm laser. Double in situ hybridization was carried out as

A

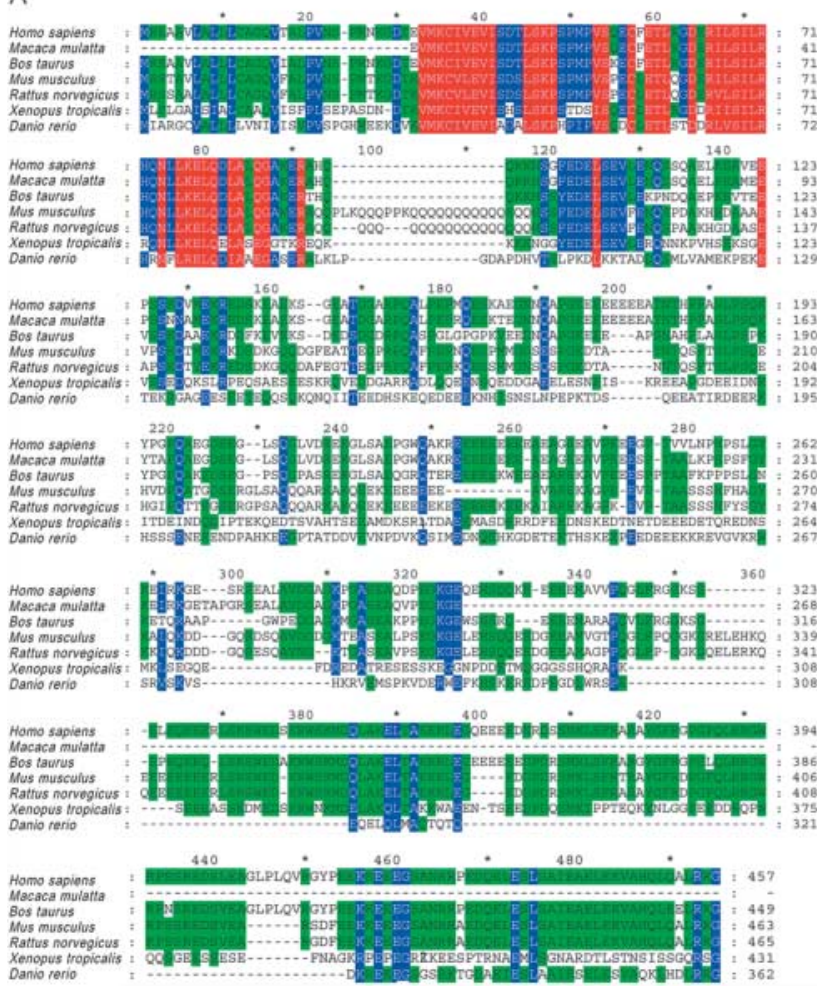

described previously (Jowett 2001). Stained embryos were postfixed in 4\% PFA and washed for 15 min twice in PBST $(0 \cdot 1 \%$ Triton $\mathrm{X}-100$ in PBS). This was followed by tissue clarification in 50\% glycerol in PBS. Embryos were mounted on glass slides and photographed under stereo microcopy (NIKON) and inverted microscope (LEICA).

\section{Results}

\section{Structural characterization of the zebrafish Chga}

Sequence of $D$. rerio Chga protein encodes 362 amino acids, and it shares $40 \%$ similarities with human CHGA, the percentages of similarities of $D$. reriochga and each species are 31\%, 40\%, 38\%, 40\%, and 36\% with Macaca mulatta, Bos taurus, Mus musculus, Rattus norvegicus, and Xenopus tropicalis respectively (Fig. 1A).D. reriochga has a conserved phylogenetic relationship among species (mentioned before) (Fig. 1B). The genomic structures of both the D. reriochga gene (25 $773 \mathrm{bp})$ and that of human (12 $144 \mathrm{bp})$ are composed of eight exons and seven introns (Fig. 1C).

B

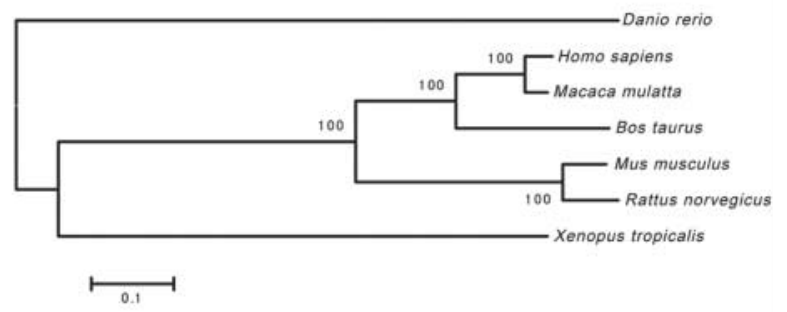

C

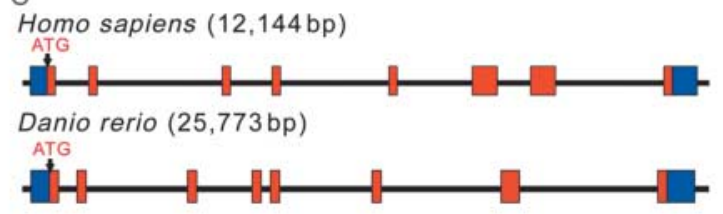

D

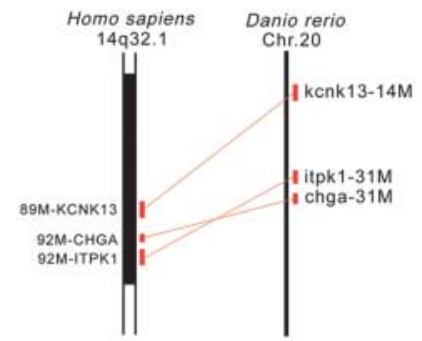

Figure 1 Homology analysis of the zebrafish Chga. (A) The amino acid sequence of the zebrafish Chga is aligned with the CHGA amino acid sequence of the Homo sapiens, Macaca mulatta, Bos taurus, Mus musculus, Rattus norvegicus, and Xenopus tropicalis. Conversed sequences are colored with four levels from primary red to secondary blue, to tertiary green and base white. (B) Phylogenetic analysis of the $\mathrm{CHGA}$ amino sequences among species. The branches are drawn so that their lengths are proportional to the evolutionary distance along that branch. The scale relates the length of a branch to the distance. (C) Genomic structure of zebrafish chga and human $C H G A$. The blue boxes indicate the $5^{\prime}$-untranslated region (5'UTR, left) and $3^{\prime}$-UTR (right), the red boxes and the lines between red boxes denote exons and introns respectively. (D) Comparison of syntenic relationships of the CHGA on human chromosome 14 (chr.14) and zebrafish chromosome 20 (chr.20). 
In humans, there are two genes in the vicinity of CHGA locus: KCNK13 and ITPK1, which together define a $3 \cdot 0 \mathrm{Mb}$ genomic region on chromosome 14q 31-32 (Fig. 1D, left). Each of the D. rerio homologs (kcnk13, chga, and itpk1) is located in the region of D. rerio chromosome 20 (Fig. 1D, right).

Spatial and temporal expression of chga during early embryonic development

The expression pattern of chga transcripts in the developing zebrafish embryos was examined by WISH with digoxigeninlabeled anti-sense RNA as a probe. Throughout two-cell stage to $12 \mathrm{hpf}$, no signals were detected (Fig. 2A), indicating that the chga transcripts were not expressed maternally. At $16 \mathrm{hpf}$, chga transcripts were first detected faintly in two specific regions; one region was in the vicinity of the otic placode, and the other in the dorsal side of trunk region (Fig. 2B and C). In the vicinity of the otic placode, three pairs of cellular clusters were detected bilaterally along the midline, which mark the positions of the trigeminal placode/ganglion, the statoacoustic ganglion, and the posterior lateral line placode/ganglion respectively (Fig. 2B). The expression of chga in the trunk was observed as two strings of beads bilaterally (Fig. 2C), and these chga-positive cells were reminiscent of the dorsal root ganglia (DRG) precursors.

At 18 and $24 \mathrm{hpf}$, the statoacoustic ganglion, the posterior lateral line placode/ganglion, and the newly formed anterior line placode expressed chga transcripts, while the expression in the trigeminal placode/ganglion was not detectable (Fig. 2D, $\mathrm{F}$ and $\mathrm{G})$. In the trunk region, the chga-expressing DRG precursors became more detectable, distributing distinctly at the ventrolateral edge of the spinal cord in each of the somatic segments (Fig. 2E and F).

At $35 \mathrm{hpf}$, the anterior line placode developed into the anterodorsal, the media, and the anteroventral lateral line ganglia (Liu et al. 2006). The chga expression signals were detected in anteroventral lateral line ganglia, besides, statoacoustic ganglion, and posterior lateral line ganglion. The signals in the DRG became stronger (Fig. $2 \mathrm{H}$ and I).

In $48 \mathrm{hpf}$ embryos, the anterodorsal, the anteroventral lateral line ganglia, and statoacoustic ganglion as well as posterior lateral line ganglion expressed the chga transcripts (Fig. 2K). However, the signals in the DRG were too weak to be seen (Fig. 2J).

WISH using the probe of sense chga mRNA was performed as a negative control; no positive signal was detected at any stage of embryogenesis (Fig. 2L).

Positional relationship of developmental expression of CHGA related to other interrenal-, pronephros-, and sympathoadrenal neuron/chromaffin cells expressing genes

As a result, the interrenal primordia were marked by $s c c$ on both sides of the notochord in the third somite at $24 \mathrm{hpf}$ (Fig. 3A), then assembled toward the right of the notochord at
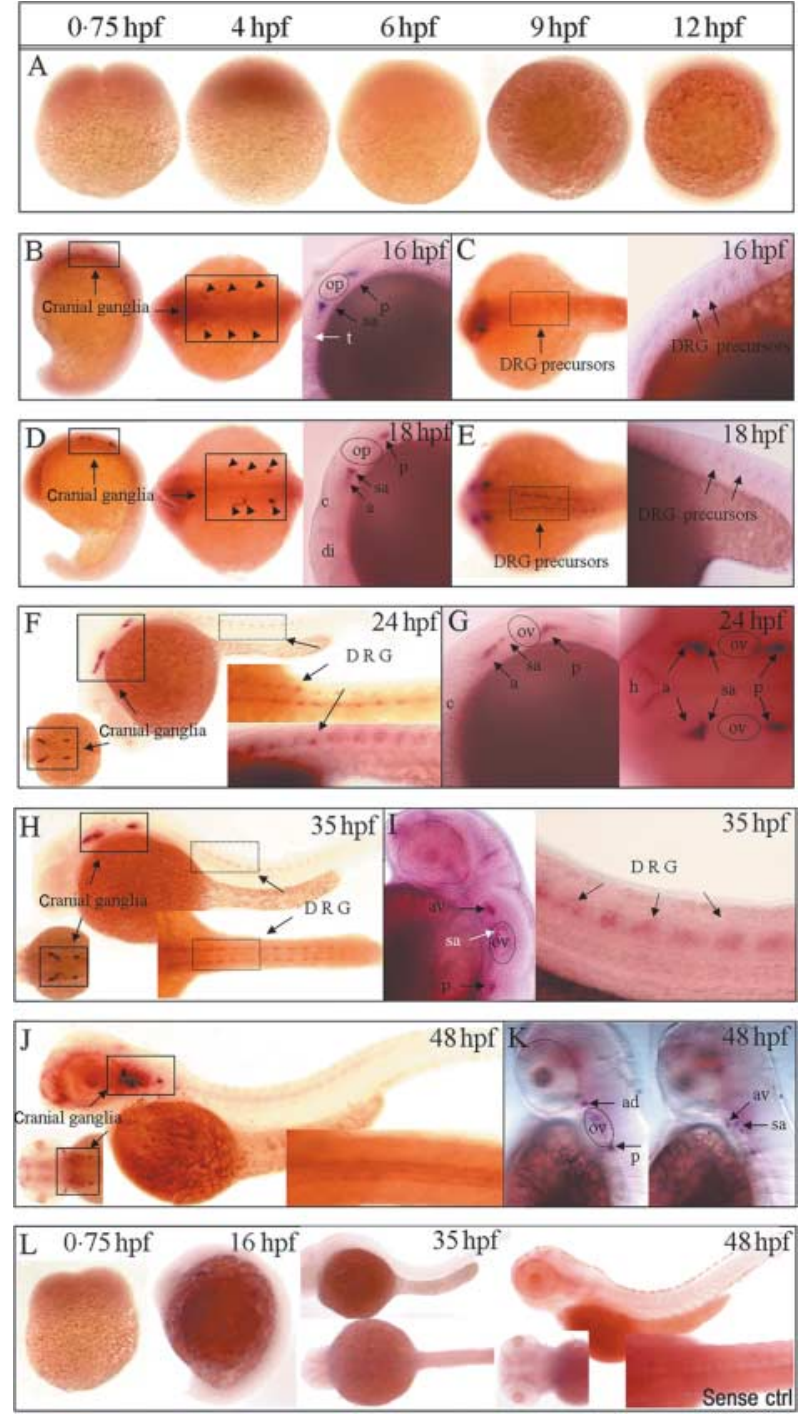

Figure 2 Spatio-temporal expression of chga in embryogenesis. (A) Lateral views of the whole-mount embryos from 0.75 to $12 \mathrm{hpf}$. (B and D) Lateral, dorsal view, and higher magnification in 16 and 18 hpf embryos. ( $C$ and $\mathrm{E}$ ) The dorsal view and the magnification lateral view of the trunk. (F, H, and J) Lateral views of the wholemount embryo, the dorsal head, and the trunk (insets) at 24, 35, and $48 \mathrm{hpf}$ respectively. (G) Higher magnification of the hindbrain (boxed part in Fig. 2F). (I) Higher magnification of the head and the trunk (boxed part in Fig. $2 \mathrm{H}$ ). (K) Higher magnification of the head (boxed part in Fig. 2J) with different focuses. (L) WISH using sense chga mRNA probe as negative control at several stages. t, trigeminal placode/ganglion; a, anterior line placode; op, otic placode; $\mathrm{p}$, posterolateral line placode/ganglion; DRG, dorsal root ganglia; sa, statoacoustic ganglion; c, cerebellum; di, diencephalon; $h$, hindbrain; av, anteroventral lateral line ganglion; ad, anterodorsal lateral line ganglion. The white arrow refers to the signals out of the focus; the otic placode (op) and the otic vesicle (ov) are outlined with dashed lines. LC, locus coeruleus; AAN, arch-associated neurons; chr, chromaffin cell; ent, enteric neurons; sym, sympathetic neurons; MC, medulla catecholaminergic cluster; Me, mesencephalon; Rha, rhombencephalon anterior part; $\mathrm{Di}$, diencephalon. 


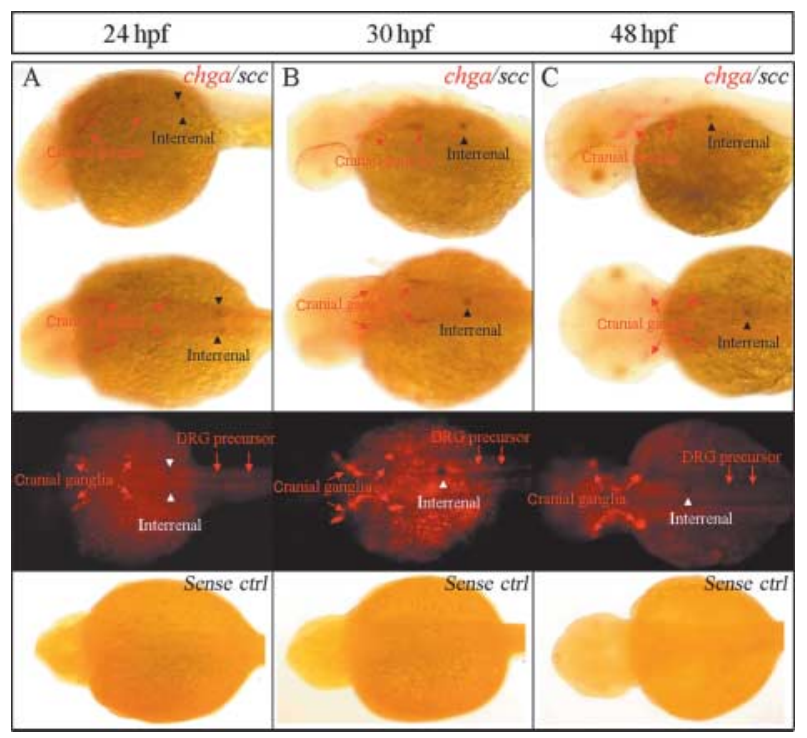

Figure 3 The distributions of the chga- and scc-expressing cells in embryogenesis. (A, B, and C) The double staining of chga (red) with sCC (dark blue) at 24,30 , and $48 \mathrm{hpf}$. From top to bottom, the lateral view (anterior facing left), the dorsal view (anterior facing left), the dorsal view under the TITC (tetraethylrhodamine isothiocyanate) filter, and the negative control with sense mRNA chga and scC probes. Arrows indicate the cranial ganglia and arrowheads indicate the interrenal.

30 and $48 \mathrm{hpf}$ (Fig. 3B and C), as described previously (Hsu et al. 2003). The pronephric primordia marked by $w t 1$ distributed on both sides of the notochord straddling over the second and third somites at 24 and $30 \mathrm{hpf}$ (Fig. 4A and B), and

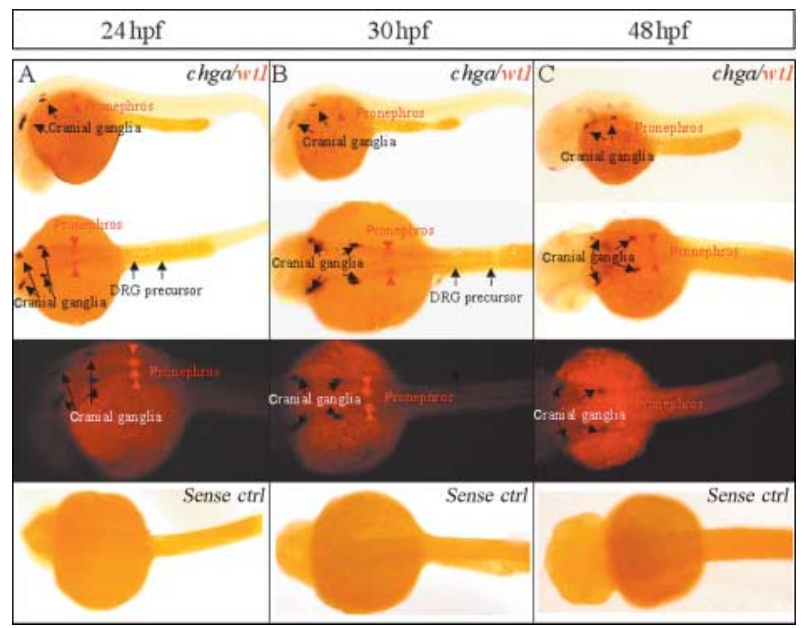

Figure 4 The distributions of the chga- and wt1-expressing cells in embryogenesis. (A, B, and C) The double staining of chga (dark blue) with wt1 (red) at 24, 30, and $48 \mathrm{hpf}$. From top to bottom, the lateral view (anterior facing left), the dorsal view (anterior facing left), the dorsal view under the TITC filter, and the negative control with sense mRNA chga and wt 1 probes. Arrows indicate the cranial ganglia and the DRG arrowheads indicate the pronephros. became spatially closer at $48 \mathrm{hpf}$ (Fig. 4C). The chgaexpressing cells were distributed in the cranial ganglia and the DRG at 24-48 hpf. Thus, our results revealed that the chga-positive cells did not co-express with either the $s c-$ or $w t 1$-positive cells. In other words, there are no chga expressed in the interrenal/pronephros during the early embryonic stages. The $d b h$-expressing cells were detected in locus coeruleus (LC) and arch-associated neurons (AAN), and they did not co-localize with the chga-expressing cells (Fig. 5).

Since we did not witness the co-expression of chga with $d b h$, $s c c$, and $w t 1$ at $48 \mathrm{hpf}$, we further evaluated the co-localization at later stages $(3,4$, and $7 \mathrm{dpf})$. At 3 and $4 \mathrm{dpf}$, the cranial ganglia (marked by chga) remained the same as observed at $48 \mathrm{hpf}$; however, chga transcripts were undetectable in the DRG (Fig. 6A and E). The $d b h$-labeled cells expressed in several areas: LC, medulla catecholaminergic cluster (AAN), cervical sympathetic neurons (sym), medulla catecholaminergic cluster (MC), and enteric neurons (ent). Some $d b h-$ expressing cells detected in the vicinity of pronephros were

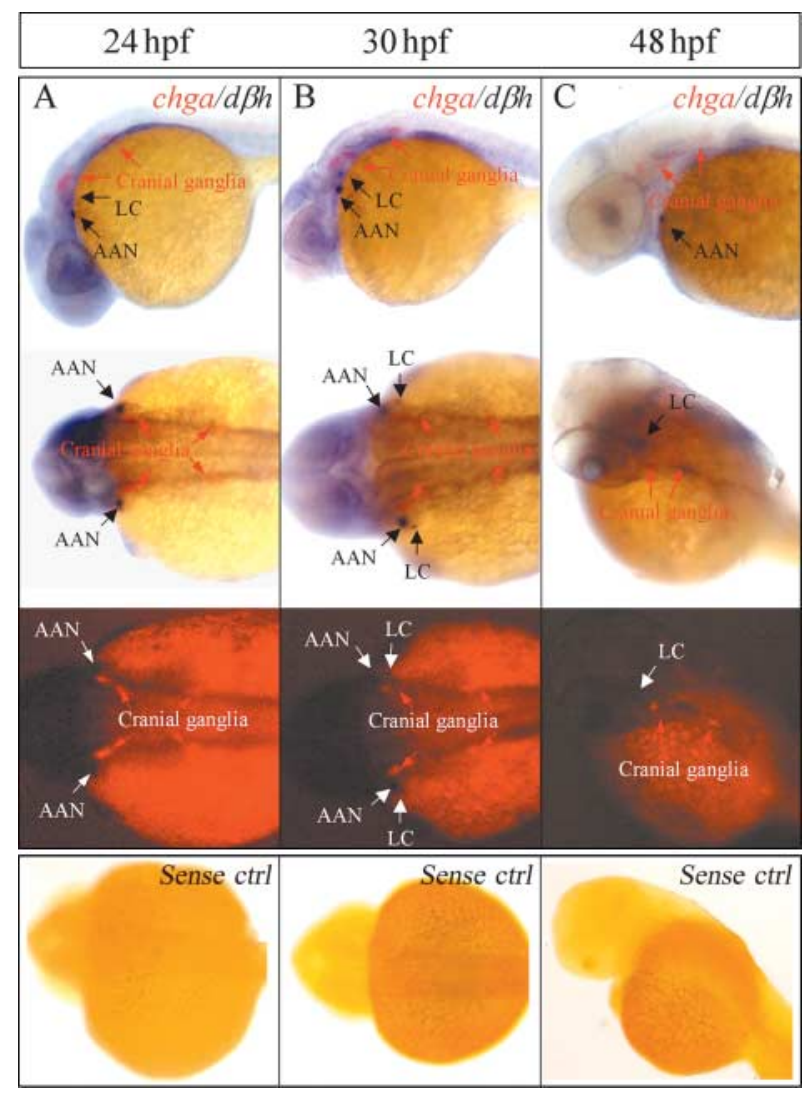

Figure 5 The distributions of the chga- and $d b h$-expressing cells in embryogenesis. (A, B, and C) The double staining of chga (red) with $d b h$ (dark blue) at 24,30 , and $48 \mathrm{hpf}$. From top to bottom the lateral view (anterior facing left), the dorsal view (anterior facing left), the dorsal view under the TITC filter (C gives the oblique view from lateral shown making the staining clear), and the negative control with sense mRNA chga and $d b h$ probes. LC, locus coeruleus; AAN, arch-associated neurons. 


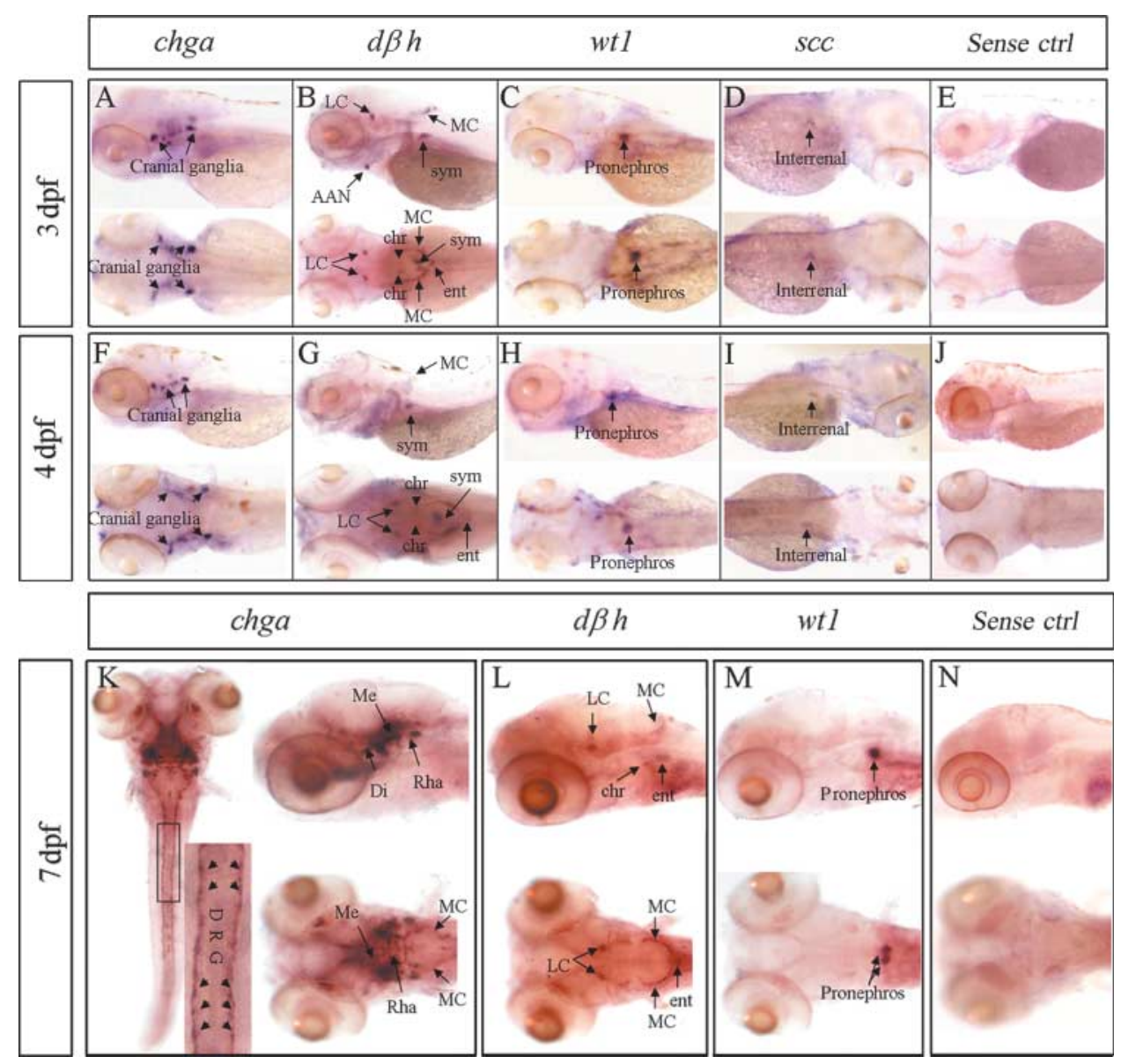

Figure 6 The distributions of chga, $d b h, w t 1$, and $s c c$ at 3, 4, and $7 \mathrm{dpf}$ in zebrafish. The chga, $d b h, w t 1$, and sCC expressions in $3 \mathrm{dpf}(\mathrm{A}-\mathrm{D}), 4 \mathrm{dpf}(\mathrm{F}-\mathrm{I})$, and $7 \mathrm{dpf}(\mathrm{K}-\mathrm{M})$ embryos. Lateral view and dorsal view in each panel. The dorsal view of the whole-mount embryo and the dorsal trunk (inset) labeled by chga are added in (K). WISH using sense chga probe as negative control (E, J, and N).

considered to be chromaffin cells (Chai et al. 2003; Fig. 6B and $\mathrm{F}$ ). The bilateral wt1-positive pronephros have fused together to become a single cellular cluster (Fig. 6C and G). The interrenal gland cells (marked by scc, Fig. 6D and $\mathrm{H}$ ) remained the same as observed at $48 \mathrm{hpf}$. At $7 \mathrm{dpf}$, it is intriguing to find that abundant chga transcripts were detected in the diencephalon, mesencephalon and anterior rhombencephalon, and the DRG (Fig. 6I). The $d b h$-labeled cells were concentrated in the LC, medulla catecholaminergic cluster (MC), enteric neurons (ent), and chromaffin cells (Fig. 6J). The $w t 1$ marked the region of the pronephros diverging into two symmetrical cellular clusters separated by the midline (Fig. 6K). The WISH using sense chga probe was performed as negative control (Fig. 6E, J and $\mathrm{N}$ ).

\section{Discussion}

We have obtained the cDNA sequences encoding the chga of zebrafish (D. rerio) from the web site (http://www.ncbi. nlm.nih.gov). Based on conserved gene structure, phylogenetic analysis, and syntenic relationship, we identified the ortholog of mammalian CHGA gene in zebrafish.

There were several studies on the early expression of CHGA in other animals. CHGA protein was found in chromaffin cells through all stages in the developing porcine and bovine adrenal medulla (Wang et al. 1994, Totzauer et al. 1995). CHGA and its derived peptides were also detected in the gastro-entero-pancreatic system of rat and porcine (Kent \& Coupland 1989), as well as in the secretory-specific cells of the hypophyseal pars tuberalis of chicken (Kameda et al. 1998). At high levels of Chga, mRNAs have been already selectively traced in the intermediate cortex at gestational day 16 in the rat brain (Mahata et al. 1993). To our knowledge, our study is the first report of the spatio-temporal expression of chga in fish.

As mentioned above, the zebrafish has the advantage of being able to trace the spatio-temporal expression of genes during early embryo development. We used the method of WISH to investigate the dynamic expression pattern during 
the serial development stages and obtained a relatively integrated picture of the chga expression pattern. Our result showed that, chga-expressing cells distributed in cranial ganglia and DRG during embryogenesis (Fig. 2) and then spread throughout in the brain and specifically in DRG (Fig. 6A). It has been already proven that the cranial ganglia derives from neural crest (Stewart et al. 2006), our result suggested the possibility that the chga-expression cells were derived from the neural crest cells and imply a possible role for chga in nervous system patterning and organogenesis.

From the previous studies, Chga has been proven to induce an activated phenotype in microglial cells that lead to neuronal apoptosis by using cultured rodent microglia (Ciesielski-Treska et al. 1998, Ulrich et al. 2002). Besides, chga transcription and gene expression inhibited cell growth in the anterior pituitary-derived cell lines and decreased the rate of tumor growth in severe combined immunodeficiency disease mice (Stilling et al. 2005). The mutant null mice for the Chga were viable and fertile and had no obvious developmental abnormalities, and their neural and endocrine functions are not grossly impaired. The authors contribute this to the compensation of the other granin family members (Hendy et al. 2006). Anyway, the specific expression of chga in zebrafish nervous system may give us clues to pay attention to relationship between the chga and nerve development.

As well known, CHGA is named for its first discovery in the chromaffin cells of adrenal medulla. The chromaffin cells are confirmed to localize in the pronephros of adult zebrafish together with the interrenal cells (Hsu et al. 2003). However, our double whole-mount in situ hybridization results indicated that chga did not co-localize with either wt1expressing pronephros or scc-expressing interrenal during early zebrafish embryogenesis (Figs 3 and 4). Besides, the results also suggested that the chga-expressing cells and the $d b h$-expressing cells might have different developmental origins (Fig. 5), and there were no chga transcripts detected within the pronephros by $7 \mathrm{dpf}$ (Fig. 6).

It has been shown that the protein products of three genes, tyrosine hydroxylase $(t h), d b h$, and phenylethanolamine-nmethyltransferase (pnmt), are required for CA synthesis, and pnmt is the most specific enzyme for converting the norepinephrine to epinephrine in the chromaffin cells of adrenal medulla (Wong 2003). Unfortunately, we are unable to detect any pnmt-expressing cells in the embryos using our current WISH protocols (Supplementary Figure 1, see Supplementary data in the online of version of the Journal of Endocrinology at http://joe.endocrinology-journals.org/ content/vol198/issue3/). Furthermore, no morphologically recognized chromaffin cells were found in the Giemsa-stained serial sections of $7 \mathrm{dpf}$ embryos (data not shown). However, the RT-PCR results did show that the pnmt transcripts can be detected in the $7 \mathrm{dpf}$ embryos, although the levels of expression were relatively low compared with th and $d b h$ (Supplementary Figure 2, see Supplementary data in the online of version of the Journal of Endocrinology at http:// joe.endocrinology-journals.org/content/vol198/issue3/).
Taken together, the results suggested that the zebrafish may have chromaffin cells, but can not be detected by pnmt WISH method likely due to either the scarcity of chromaffin cells or the low levels of pnmt transcripts during early embryogenesis. Besides, the failure to detect the pnmt transcripts in $d b h$ expressing cells may be attributed to their functional immaturity during early embryogenesis, which warrants further study.

\section{Declaration of Interest}

The authors declare that there is no conflict of interest that could be perceived as prejudicing the impartiality of the research reported.

\section{Funding}

This work was supported by Shanghai Leading Academic Discipline Project (Y0204), Shanghai Committee of Science and Technology (O64119629) and National Natural Science Foundation of China (30525019 for W Q W and 30725037 for G N), Hundred Scholars Award of Chinese Academy of Science ( $\mathrm{T}$ X L)

\section{Acknowledgements}

We thank Dr Vladimir Korzh for the $d b h$ plasmid and Prof. Chung Bon-chu for the scc plasmid.

\section{References}

Chai C, Liu YW \& Chan WK 2003 Ff1b is required for the development of steroidogenic component of the zebrafish interrenal organ. Developmental Biology 260 226-244.

Ciesielski-Treska J, Ulrich G, Taupenot L, Chasserot-Golaz S, Corti A, Aunis D \& Bader MF 1998 Chromogranin A induces a neurotoxic phenotype in brain microglial cells. Journal of Biological Chemistry 273 14339-14346.

Grassi Milano E, Basari F \& Chimenti C 1997 Adrenocortical and adrenomedullary homologs in eight species of adult and developing teleosts: morphology, histology, and immunohistochemistry. General and Comparative Endocrinology 108 483-496.

Hendy GN, Li T, Girard M, Feldstein RC, Mulay S, Desjardins R, Day R, Karaplis AC, Tremblay ML \& Canaff L 2006 Targeted ablation of the chromogranin a (Chga) gene: normal neuroendocrine dense-core secretory granules and increased expression of other granins. Molecular Endocrinology 20 1935-1947.

Hsu HJ, Lin G \& Chung BC 2003 Parallel early development of zebrafish interrenal glands and pronephros: differential control by wt 1 and ff1b. Development 130 2107-2116.

Jowett T 2001 Double in situ hybridization techniques in zebrafish. Methods 23 345-358.

Kameda Y, Miura M \& Ohno S 1998 Localization and development of chromogranin A and luteinizing hormone immunoreactivities in the secretory-specific cells of the hypophyseal pars tuberalis of the chicken. Histochemistry and Cell Biology 109 211-222.

Kent C \& Coupland RE 1989 Localisation of chromogranin A and B, met-enkephalin-arg6-gly7-leu8 and PGP9.5-like immunoreactivity in the developing and adult rat adrenal medulla and extra-adrenal chromaffin tissue. Journal of Anatomy 166 213-225.

Kim T \& Loh YP 2005 Chromogranin A: a surprising link between granule biogenesis and hypertension. Journal of Clinical Investigation 115 1711-1713. 
Kimmel CB, Ballard WW, Kimmel SR, Ullmann B \& Schilling TF 1995 Stages of embryonic development of the zebrafish. Developmental Dynamics 203 253-310.

Liu Q, Liu B, Wilson AL \& Rostedt J 2006 Cadherin-6 message expression in the nervous system of developing zebrafish. Developmental Dynamics 235 272-278.

Mahata M, Mahata SK, Fischer-Colbrie R \& Winkler H 1993 Ontogenic development and distribution of mRNAs of chromogranin A and B, secretogranin II, p65 and synaptin/synaptophysin in rat brain. Brain Research. Developmental Brain Research 76 43-58.

McGonnell IM \& Fowkes RC 2006 Fishing for gene function - endocrine modelling in the zebrafish. Journal of Endocrinology 189 425-439.

Reid SG, Fritsche R \& Jonsson AC 1995 Immunohistochemical localization of bioactive peptides and amines associated with the chromaffin tissue of five species of fish. Cell and Tissue Research 280 499-512.

Song HD, Sun XJ, Deng M, Zhang GW, Zhou Y, Wu XY, Sheng Y, Chen Y, Ruan Z, Jiang CL et al. 2004 Hematopoietic gene expression profile in zebrafish kidney marrow. PNAS 101 16240-16245.

Stewart RA, Arduini BL, Berghmans S, George RE, Kanki JP, Henion PD \& Look AT 2006 Zebrafish foxd3 is selectively required for neural crest specification, migration and survival. Developmental Biology 292 174-188.

Stilling GA, Bayliss JM, Jin L, Zhang H \& Lloyd RV 2005 Chromogranin A transcription and gene expression in Folliculostellate (TtT/GF) cells inhibit cell growth. Endocrine Pathology 16 173-186.
Taupenot L, Harper KL \& O'Connor DT 2003 The chromograninsecretogranin family. New England Journal of Medicine 348 1134-1149.

Tischler AS, Powers JF \& Alroy J 2004 Animal models of pheochromocytoma. Histology and Histopathology 19 883-895.

Totzauer I, Amselgruber W, Sinowatz F \& Gratzl M 1995 Early expression of chromogranin A and tyrosine hydroxylase during prenatal development of the bovine adrenal gland. Anatomy and Embryology 191 139-143.

Ulrich G, Ciesielski-Treska J, Taupenot L \& Bader MF 2002 Chromogranin A-activated microglial cells induce neuronal apoptosis. Annals of the New York Academy of Sciences 971 560-562.

Unsicker K, Huber K, Schutz G \& Kalcheim C 2005 The chromaffin cell and its development. Neurochemistry Research 30 921-925.

Wang JM, De Ridder EF, De Potter WP \& Weyns AL 1994 Localization of neurokinin A and chromogranin A immunoreactivity in the developing porcine adrenal medulla. Histochemical Journal 26 431-436.

Wong DL 2003 Why is the adrenal adrenergic? Endocrine Pathology 14 25-36.

Received in final form 18 June 2008

Accepted 27 June 2008

Made available online as an Accepted Preprint

27 June 2008 